\title{
Extended Kalman Filtering for the Modeling and Analysis of ICG Pharmacokinetics using NIR Optical Methods
}

\author{
Burak Alacam $^{a}$, Birsen Yazıcı ${ }^{a}$, Xavier Intes ${ }^{b}$, and Britton Chance ${ }^{c}$ \\ ${ }^{a}$ Electrical, Computer and Systems Engineering Dept., Rensselaer Polytechnic Institute, USA \\ ${ }^{b}$ ART Advanced Research Technologies Inc., Canada \\ ${ }^{c}$ Department of Biochemistry and Biophysics, University of Pennsylvania, USA
}

\begin{abstract}
A number of studies indicate that compartmental modeling of indocyanine green (ICG) pharmacokinetics, as measured by near infrared (NIR) techniques, may provide diagnostic information for tumor differentiation. However, compartmental parameter estimation is a highly non-linear problem with limited data available in a clinical setting. Furthermore, pharmacokinetic parameter estimates show statistical variation from one data set to another. Thus, a systematic and robust approach is needed to model, estimate and quantify ICG pharmacokinetic parameters. In this paper, we propose to model ICG pharmacokinetics in extended Kalman filtering (EKF) framework. EKF effectively models multiple-compartment and multiple-measurement systems in the presence of measurement noise and uncertainties in model dynamics. It provides simultaneous estimation of pharmacokinetic parameters and ICG concentrations in each compartment. Moreover, recursive nature of the Kalman filter estimator potentially allows real time monitoring of time varying pharmacokinetic rates and concentration changes in different compartments. We tested our approach using the ICG concentration data acquired from four Fischer rats carrying adenocarcinoma tumor cells. Our study indicates that EKF model may provide additional parameters that may be useful for tumor differentiation.
\end{abstract}

Keywords: Extended Kalman Filter, Indocyanine Green, Compartmental Analysis, Pharmacokinetics

\section{INTRODUCTION}

NIR diffuse optical imaging and spectroscopy methods provide quantitative functional information that can not be obtained by the conventional radiological methods. ${ }^{1-4}$ NIR techniques can provide in vivo measurements of oxygenation and vascularization state, uptake and release of optical contrast agents and chromophore concentrations with high sensitivity. There is considerable evidence that NIR diffuse optical techniques in conjunction with optical contrast agents have the potential to characterize angiogenesis and to differentiate between malignant and benign tumors. ${ }^{5,6}$

Among many optical contrast agents, ICG is the only agent approved for human use. In NIR measurements, the presence of ICG within an imaging volume results in an increased signal that can be observed over the course of the experiment. Study of the time kinetics of ICG concentration curves may provide physiologically relevant information for tumor differentiation. Specifically, cancerous tissue types are expected to show high and fast uptake due to proliferation of "leaky" angiogenetic microvessels, while normal and fatty tissue show little uptake.

A number of research groups reported compartmental modeling of ICG time-kinetic measurements using NIR methods for tumor diagnosis in animal and human subjects. ${ }^{7-9}$ Parameters related to capillary permeability were suggested as malignancy indicators. Compartmental model is a mathematical description of the concentrations of contrast agents in which each compartment represents kinetically distinct tissue type. It consists of a set of coupled ordinary partial differential equations (ODE) and a measurement model. Coefficients of the ODE's are the physiological parameters of interest that represent rates of exchange between different compartments. These parameters are non-linearly related to the total concentration of ICG measured by NIR methods. Furthermore, concentration of ICG in each compartment can not be directly measured by NIR techniques, making the pharmacokinetic parameter estimation a highly non-linear problem.

\footnotetext{
Further author information: Send correspondence to Birsen Yazici, e-mail: yazici@ecse.rpi.edu
} 
Current methods of ICG pharmacokinetic modeling involves curve fitting methods and various techniques for solving differential equations. Gurfinkel et. al. presented a two-compartmental model for ICG kinetics and estimated model parameters. ${ }^{7}$ The measurements were obtained using a frequency domain photon migration system coupled with a charge-coupled device. The pharmacokinetic parameters are estimated for each pixel based on a curve fitting method. This study indicates that model parameters show no difference in the ICG uptake rates between normal and diseased tissue. Cuccia et al. presented a study of the dynamics of ICG in an adenocarcinoma rat tumor model. ${ }^{8}$ A two-compartmental model describing the ICG dynamics is used to quantify physiologic parameters related to capillary permeability. The ICG concentration curves were fitted to the compartmental model using a non-linear least squares Levenberg-Marquart algorithm. It was shown that different tumor types have different capillary permeability rates. Intes et. al. presented the uptake of ICG by breast tumors using a continuous wave diffuse optical tomography apparatus. ${ }^{9}$ A two-compartment model is used to analyze the pharmacokinetics of ICG. A curve fitting algorithm namely, nonlinear Nelder-Mead simplex search, is used to estimate the pharmacokinetic parameters. This study shows that the malignant cases exhibit slower rate constants (uptake and outflow) as compared to healthy tissue.

Clearly, these studies indicate that ICG pharmacokinetic parameters have the potential to provide diagnostic information. However, due to highly non-linear nature of the estimation problem, variation in parameters values from one data set to another, and sparse data available in a clinical setting, a systematic and robust approach is needed to estimate ICG pharmacokinetic parameters. In this paper, we propose to model ICG pharmacokinetics in an extended Kalman filtering (KF) framework. KF is a widely used optimal recursive modeling and estimation method. It effectively models multiple compartments, and measurement systems governed by coupled ordinary differential equations in the presence of measurement noise and uncertainties in the compartmental model dynamics. Extended Kalman filter (EKF) provides a recursive estimation of not only the permeability rates but also the ICG concentrations in each compartment, which is not directly accessible in vivo by means of NIR techniques. EKF can also successfully accommodate any information available about the initial conditions of the permeability rates into the estimation procedure. Furthermore, estimation is recursive that is, the estimates are updated as new measurements arrive. As a result, EKF based modeling allows potentially real-time monitoring of ICG pharmacokinetic parameters and time-varying ICG concentrations in different compartments.

We tested our approach using the ICG concentration data acquired from four Fischer rats carrying adenocarcinoma tumor cells. Compartmental model parameters and concentrations in different compartments are estimated. Model parameters were used to differentiate between two different stages of tumor. We observed that the permeability rates are higher in advanced tumor stages. Additionally, we observed that the concentration curve of ICG is higher in the EES compartment in advanced tumor stages. This suggests that the ratio of the peak value of the ICG concentrations in different compartments may be a useful parameter to differentiate tumors.

The paper is organized as follows: In Section 2, we present the compartmental modeling of ICG pharmacokinetics. In Section 3, we present modeling and estimation of ICG pharmacokinetics parameters and ICG concentrations in extended Kalman filtering framework. In Section 4, we present experimental results obtained from Fischer rat data. Finally, Section 5 concludes our discussion.

\section{COMPARTMENTAL MODELING OF ICG PHARMACOKINETICS}

\subsection{Indocyanine Green}

ICG is an optical dye commonly used in retinopathy and hepatic diagnostics. Given its low toxicity and FDA approval, it has recently been utilized as a blood pooling agent for the detection and diagnosis of cancerous tumors by means of NIR optical methods. The absorption peak of ICG is $805 \mathrm{~nm}$ and the fluorescence peak is at $830 \mathrm{~nm}$. ICG has strong affinity for blood proteins. In plasma, ICG is near-completely bound, primarily to albumin. As a result, its in vivo kinetics are similar to those of a $70 \mathrm{kD}$ molecule although it has a molecular weight of about $700 \mathrm{D}$. Given its effective size, it is expected that little will exit the vasculature. However, in certain tumor lines, the poorly developed capillary bed allows extravasation of albumin and therefore ICG. ${ }^{10-14}$

ICG is eliminated from the body primarily through the bile (the fluid flowing to the intestines to aid in digestion and removal of waste). ICG outside of the circulatory system is not available for removal until it returns 


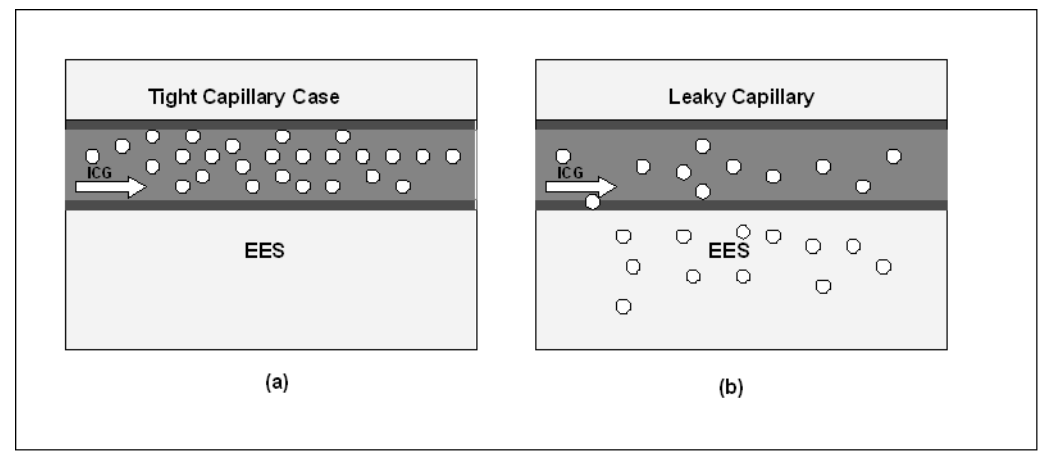

Figure 1. (a) ICG flow in tight capillary of normal vessel, (b) ICG flow in permeable capillary of tumor tissue .

to the system. The kinetics of this transition offers a potential means to non-invasively assess the leakiness of large molecules from the microvasculature; this permeability is a characteristic of the poorly developed vasculature observed in angiogenesis. The increase in local microvasculature density also increases the magnitude of the photon density from inter-capillary ICG. Therefore pharmacokinetic analysis of ICG may provide information for cancer diagnosis and staging. ${ }^{8,9}$

\subsection{Compartmental Modeling of ICG Pharmacokinetics}

Compartmental modeling allows relatively simple and effective representation of complex biological responses due to drugs or contrast agents. A region of interest is assumed to consist of a number of compartments, generally representing a volume or a group of similar tissues into which the contrast agent is distributed. The concentration change in a specific compartment is modeled as a result of the exchange of contrast agent between connected compartments. These changes are modeled by a collection of coupled ODEs, each equation describing the time change dictated by the biological laws that govern the concentration exchanges between interacting compartments. ${ }^{15-18}$

There are some differences in the delivery of ICG between normal vasculature and cancer vasculature. In normal tissue, ICG acts as a blood flow indicator in tight capillaries of normal vessel. However in tumor, ICG may act as a diffusible (extravascular) flow in leaky capillary of cancer vessels. Additionally, The ICG leaks slowly from plasma into extracellular extravascular space (EES) when capillary permeability increases. The permeability is expected to increase as the malignancy advances. Figure 1 (a) and (b) illustrates the ICG flow for healthy and malignant tissue, respectively.

In this paper, the tumor region is assumed to be composed of two compartments; namely, the plasma and EES. ${ }^{8,19,20}$ Thus, a two-compartment model is chosen to represent the time-kinetics of ICG concentrations. Figure 2 shows the two-compartment model for ICG kinetics. $C_{p}$ and $C_{e}$ represent the ICG concentrations in the plasma and EES, respectively. The parameters $k_{i n}$ and $k_{\text {out }}$ govern the leakage into and the drainage out of the EES. The parameter $k_{\text {elm }}$ describes the ICG elimination from the body through kidneys and livers. The parameters $v_{p}$ and $v_{e}$ are the plasma and EES volume fractions, respectively.

The leakage into and the drainage out of the EES is given by:

$$
\frac{d C_{e}(t)}{d t}=-k_{\text {out }} C_{e}(t)+k_{i n} C_{p}(t)
$$

The leakage into and the drainage out of plasma is given by:

$$
\frac{d C_{p}(t)}{d t}=-\left(k_{\text {in }}+k_{\text {elm }}\right) C_{p}(t)+k_{\text {out }} C_{e}(t)
$$

The parameters $k_{i n}, k_{\text {out }}$, and $k_{\text {elm }}$ have a unit of $s e c^{-1}$. They are defined as the permeability surface area products given by $P S \rho$, where $P$ is the capillary permeability constant, $S$ is the capillary surface area, and $\rho$ is 


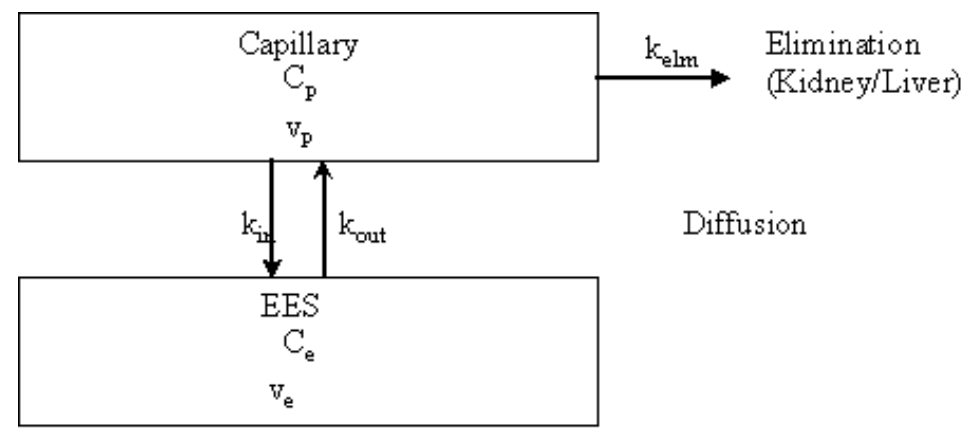

Figure 2. Representation of two compartment ICG pharmacokinetic model.

the tissue density. We consider transcapillary leakage to occur only at the tumor site. We also assume that a small perturbation of the global plasma concentration does not affect the bulk removal.

Actual bulk ICG concentration in the tissue measured by NIR spectroscopy, $m(t)$, is a linear combination of plasma and EES ICG concentrations:

$$
m(t)=v_{p} C_{p}(t)+v_{e} C_{e}(t),
$$

where $v_{p}, v_{e}, C_{e}$, and $C_{p}$ are as defined above.

\section{EXTENDED KALMAN FILTERING FOR THE ICG PHARMACOKINETICS}

\subsection{State-space representation of ICG time-kinetics}

Coupled differential equations resulting from the two-compartment model of the ICG pharmacokinetics can be expressed in state-space representation:

$$
\begin{gathered}
{\left[\begin{array}{c}
\dot{C}_{e}(t) \\
\dot{C}_{p}(t)
\end{array}\right]=\left[\begin{array}{cc}
-k_{\text {out }} & k_{\text {in }} \\
k_{\text {out }} & -\left(k_{\text {in }}+k_{\text {elm }}\right)
\end{array}\right]\left[\begin{array}{l}
C_{e}(t) \\
C_{p}(t)
\end{array}\right]+\boldsymbol{\omega}(t),} \\
m(t)=\left[\begin{array}{ll}
v_{e} & v_{p}
\end{array}\right]\left[\begin{array}{c}
C_{e}(t) \\
C_{p}(t)
\end{array}\right]+\eta(t)
\end{gathered}
$$

where $\boldsymbol{\omega}(t)$ and $\eta(t)$ are uncorrelated zero mean Gaussian processes with covariance matrix $Q$, and variance $\sigma^{2}$, respectively.

The closed form of the continuous time state-space representation is given by:

$$
\begin{gathered}
d \mathbf{C}(t)=\kappa(\boldsymbol{\alpha}) \mathbf{C}(t) d t+\boldsymbol{\omega}(t) d t, \\
m(t)=V(\boldsymbol{\alpha}) \mathbf{C}(t)+\eta(t) .
\end{gathered}
$$

In equation (5), $\mathbf{C}(t)$ denotes the concentration vector with elements $C_{e}(t)$, and $C_{p}(t) ; \kappa(\boldsymbol{\alpha})$ is the $2 \times 2 \mathrm{KF}$ system matrix, $V(\boldsymbol{\alpha})$ is the $1 \times 2 \mathrm{KF}$ measurement matrix as defined in equation (4) and $\boldsymbol{\alpha}$ is the parameter vector

$$
\boldsymbol{\alpha}=\left[\begin{array}{lllll}
k_{\text {out }} & k_{\text {in }} & k_{\text {elm }} & v_{e} & v_{p}
\end{array}\right] .
$$

The ICG measurements in equation (5) are collected at discrete time instances, $t=k T, k=0,1, \ldots$, where $T$ is the sampling period. Therefore, the continuous model described in equations (5) has to be discretized. To simplify our notation, we shall use $\mathbf{C}(k)=\mathbf{C}(k T)$ and $\mathbf{m}(k)=\mathbf{m}(k T)$. The discrete KF system and observation models are given as follows: 


$$
\begin{gathered}
\mathbf{C}(k+1)=\kappa_{d}(\boldsymbol{\alpha}) \mathbf{C}(k)+\boldsymbol{\omega}(k) \\
\mathbf{m}(k)=V_{d}(\boldsymbol{\alpha}) \mathbf{C}(k)+\eta(k),
\end{gathered}
$$

where $\kappa_{d}(\boldsymbol{\alpha})=e^{\kappa(\boldsymbol{\alpha})}$ is the discrete time KF system matrix and $V_{d}(\boldsymbol{\alpha})=V(\boldsymbol{\alpha})$ is the discrete KF measurement matrix. $\boldsymbol{\omega}(k)$ and $\eta(k)$ are zero mean Gaussian white noise processes with covariances matrix $Q_{d}$ and variance $\sigma_{d}^{2}$, respectively. A general discussion on the discretization of state-space models can be found in various Kalman filtering books, see for example. ${ }^{21}$

An explicit form for the discrete KF model is given as follows:

$$
\begin{gathered}
{\left[\begin{array}{c}
C_{e}(k+1) \\
C_{p}(k+1)
\end{array}\right]=\left[\begin{array}{ll}
\tau_{11} & \tau_{12} \\
\tau_{21} & \tau_{22}
\end{array}\right]\left[\begin{array}{l}
C_{e}(k) \\
C_{p}(k)
\end{array}\right]+\boldsymbol{\omega}(k)} \\
\mathbf{m}(k)=\left[\begin{array}{ll}
v_{e} & v_{p}
\end{array}\right]\left[\begin{array}{l}
C_{e}(k) \\
C_{p}(k)
\end{array}\right]+\eta(k),
\end{gathered}
$$

where $\tau_{i j}$ is the $i^{t h}$ row and $j^{t h}$ column entry of the system matrix $\kappa_{d}(\boldsymbol{\alpha})$. The matrix entry $\tau_{i j}$ is an exponential function of the parameters $k_{i n}, k_{\text {out }}$ and $k_{\text {elm }}$. To simplify estimation process, we shall first estimate $\tau_{i j}$ 's and then compute the pharmacokinetic parameters $k_{i n}, k_{\text {out }}$ and $k_{\text {elm }}$.

\subsection{Extended Kalman filtering for ICG pharmacokinetics}

Kalman filter provides a recursive method to estimate the states in state-space models, in which states are driven by noise and measurements are made in the presence of noise. ${ }^{2-24}$ It is one of the most widely used methods for estimation in engineering problems due to its simplicity, optimality and robustness. In the case of non-linear state-space models, the extended Kalman filter linearizes the model around the current state estimate, and then applies the KF to the resulting linear model. The EKF framework is also utilized for the joint estimation of the unknown system and/or measurement parameters and states. In a linear state-space model when both states and system parameters are unknown, the linear state-space model can be regarded as a non-linear model in which linear system parameters and states are combined to form the new states of the non-linear model. This system is then linearized and solved for the unknown states using KF framework. ${ }^{25-27}$ In our problem, we want to simultaneously estimate the states, and system and measurement parameters given in equation (8), i.e., the ICG concentrations in plasma and EES; pharmacokinetic parameters and volume fractions.

We consider a Taylor series approximation to the non-linear system function at the previous state estimates and that of the measurement function at the corresponding predicted position. This approach provides a simple and efficient method to handle the non-linearity in the new system and measurement models.

Let $\boldsymbol{\theta}(k)$ be the discrete parameter vector defined as:

$$
\boldsymbol{\theta}(k)=\left[\begin{array}{llllll}
\tau_{11} & \tau_{12} & \tau_{21} & \tau_{22} & v_{e} & v_{p}
\end{array}\right]_{k}^{T} .
$$

In EKF framework, $\boldsymbol{\theta}(k)$ is treated as a random process with the following model:

$$
\boldsymbol{\theta}(k+1)=\boldsymbol{\theta}(k)+\boldsymbol{\varsigma}(k),
$$

where $\boldsymbol{\varsigma}(k)$ is a zero mean white noise process with covariance matrix $S_{d}$.

We append the parameter vector $\boldsymbol{\theta}(k+1)$ to the ICG concentration vector $\mathbf{C}(k+1)$ to form the new non-linear state-space model given as

$$
\begin{aligned}
& {\left[\begin{array}{c}
\mathbf{C}(k+1) \\
\boldsymbol{\theta}(k+1)
\end{array}\right]=\left[\begin{array}{c}
\mathbf{K}(\boldsymbol{\theta}) \mathbf{C}(k) \\
\boldsymbol{\theta}(k)
\end{array}\right]+\left[\begin{array}{c}
\boldsymbol{\omega}(k) \\
\boldsymbol{\varsigma}(k)
\end{array}\right]} \\
& \mathbf{m}(k)=\left[\begin{array}{ll}
V_{d}(\boldsymbol{\theta}) & 0
\end{array}\right]\left[\begin{array}{c}
\mathbf{C}(k) \\
\boldsymbol{\theta}(k)
\end{array}\right]+\eta(k),
\end{aligned}
$$


where $\mathbf{K}(\theta)=\kappa_{d}(\boldsymbol{\alpha})$.

The choice of $Q_{d}, S_{d}$ and $\sigma_{d}^{2}$ is crucial to the performance EKF estimator. It was shown that if these values are selected to be less than or equal to the actual values, it leads to overconfidence in the accuracy of the estimates of the error covariance matrix. Therefore, these matrices should be regarded as tuning parameters and not as the estimates of the true covariance matrices. ${ }^{28}$

\subsection{Joint estimation of ICG concentrations, pharmacokinetic parameters, and volume fractions}

We will summarize the major steps of the EKF estimator. The following equations describe how the state estimates and error covariance matrix are updated at the $k^{\text {th }}$ time instant given all the measurements up to $(k-1)^{t h}$ time instant. For $k=1,2,3, \ldots$,

$$
\begin{aligned}
& {\left[\begin{array}{c}
\hat{C}_{e} \\
\hat{C}_{p} \\
\hat{\boldsymbol{\theta}}
\end{array}\right]_{k \mid k-1}=\left[\begin{array}{c}
\tau_{11} \hat{C}_{e}+\hat{\tau_{12}} \hat{C}_{p} \\
\tau_{21} \hat{C}_{e}+\hat{\tau_{22}} \hat{C}_{p} \\
\hat{\boldsymbol{\theta}}
\end{array}\right]_{k-1 \mid k-1}} \\
& P_{k \mid k-1}=J_{k-1} P_{k-1 \mid k-1} J_{k-1}^{T}+\left[\begin{array}{cc}
Q_{d} & 0 \\
0 & S_{d}
\end{array}\right] .
\end{aligned}
$$

The subscript $k \mid k-1$ denotes the estimate at time $k$ given all the measurements up to time $t . P_{k \mid k-1}$ is the error covariance matrix of the estimates. $J_{k-1}$ is the $8 \times 8$ Jacobian matrix of the non-linear EKF system function at time $k-1$ given by

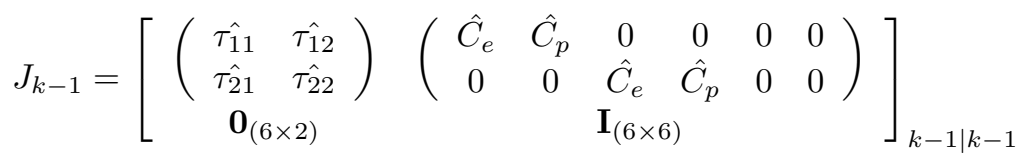

where $\mathbf{0}_{(6 \times 2)}$ is the zero matrix, and $\mathbf{I}_{(6 \times 6)}$ is the identity matrix.

The Kalman gain is given as

$$
G_{k}=P_{k \mid k-1} \Lambda^{T}\left[\Lambda P_{k \mid k-1} \Lambda^{T}+\sigma_{k}^{2}\right]^{-1},
$$

where $\Lambda$ is the vector

$$
\left[\begin{array}{llllllll}
\hat{v_{e}} & \hat{v_{p}} & 0 & 0 & 0 & 0 & \hat{C}_{e} & \hat{C}_{p}
\end{array}\right]_{k \mid k-1} .
$$

The error covariance matrix update is given by

$$
P_{k \mid k}=\left[I-G_{k} \Lambda\right] P_{k \mid k-1}
$$

where $\mathbf{I}$ is the $8 \times 8$ identity matrix.

The $k^{t h}$ step estimate of the concentrations and the parameters are obtained recursively using

$$
\left[\begin{array}{c}
\hat{C}_{e} \\
\hat{C}_{p} \\
\hat{\boldsymbol{\theta}}
\end{array}\right]_{k \mid k}=\left[\begin{array}{c}
\hat{C}_{e} \\
\hat{C}_{p} \\
\hat{\boldsymbol{\theta}}
\end{array}\right]_{k \mid k-1}+G_{k}\left(\mathbf{m}(k)-\hat{v}_{e} \hat{C}_{e}-\hat{v_{p}} \hat{C}_{p}\right)_{k \mid k-1} .
$$

Figure 3 shows the complete operation of the EKF algorithm.

The initialization of the plasma and EES concentrations, pharmacokinetic parameters, and the volume fractions plays an important role in the performance of the EKF algorithm. Theoretically, the state estimates can be initialized at the expected value of the ICG concentations, i.e. $E[\mathbf{C}(0)]$. One approach to the initialization of the parameters is to utilize the state-space presentation given in equation (7). Since $E(\mathbf{m}(0))=V_{d}(\boldsymbol{\theta}(0)) E[\mathbf{C}(0)]$, $\mathbf{m}(0)-V_{d}(\boldsymbol{\theta}(0)) E[\mathbf{C}(0)]$ is a zero mean random variable. If we express the variance of the measurement $\mathbf{m}(0)$ in 


\begin{tabular}{|c|c|}
\hline $\begin{array}{l}\text { System Model } \\
\text { Measurement Model }\end{array}$ & $\begin{array}{l}{\left[\begin{array}{l}\mathbf{C}(k+1) \\
\boldsymbol{\theta}(k+1)\end{array}\right]=\left[\begin{array}{cc}\kappa_{d}(\boldsymbol{\theta}(k)) \mathbf{C}(k) \\
\boldsymbol{\theta}(k)\end{array}\right]+\left[\begin{array}{l}\mathbf{w}(k) \\
\boldsymbol{\zeta}(k)\end{array}\right]} \\
\mathbf{m}(k)=\left[\begin{array}{ll}V_{d}(\boldsymbol{\theta}(k)) & 0\end{array}\right]\left[\begin{array}{l}\mathbf{C}(k) \\
\boldsymbol{\theta}(k)\end{array}\right]+\eta(k)\end{array}$ \\
\hline Initial Conditions & $\begin{array}{l}{\left[\begin{array}{l}\mathbf{C}(0) \\
\boldsymbol{\theta}(0)\end{array}\right]=\left[\begin{array}{c}E(\mathbf{C}(0)) \\
\boldsymbol{\theta}(0)\end{array}\right]} \\
P_{0,0}=\left[\begin{array}{cc}\operatorname{Cov}(\mathbf{C}(0)) & 0 \\
0 & S_{d}\end{array}\right]\end{array}$ \\
\hline $\begin{array}{l}\text { Kalman Gain Estimate } \\
\text { Error Covariance Matrix Estimate } \\
\text { State and Parameter Estimate }\end{array}$ & $\begin{aligned} & G_{k}=P_{k, k-1} \Lambda^{T}\left[\Lambda P_{k, k-1} \Lambda^{T}+\sigma_{\dot{d}}\right] \\
& P_{k, k}=\left[I-G_{k} \Lambda P_{k, k-1}\right. \\
& {\left[\begin{array}{c}C_{e} \\
C_{p} \\
\theta\end{array}\right]_{k}=\left[\begin{array}{c}C_{e} \\
C_{p} \\
\boldsymbol{\theta}\end{array}\right]_{k k-1}+G_{k}\left(\mathbf{m}(k)-v_{e} C_{e}-v_{p} C_{p}\right)_{k k-1} }\end{aligned}$ \\
\hline
\end{tabular}

Figure 3. The extended Kalman filter algorithm for joint concentration and pharmacokinetic parameter estimation .

terms of the variance of $\mathbf{C}(0)$ using the measurement model in equation (7), and solve for $\boldsymbol{\theta}$, we get the estimate $\hat{\boldsymbol{\theta}}(0)$ as the most appropriate value for initialization. The details of the selection of the initial values for the parameters can be found in. ${ }^{23}$

The initialization of the error covariance matrix is also important for the performance of the EKF. The error covariance matrix is the matrix which provides the information about the error bounds for the estimates. Theoretically, the initial error covariance matrix is a diagonal matrix where the diagonal entries are the initial estimates of the variance of concentrations and pharmacokinetic parameters, i.e.

$$
P_{0 \mid 0}=\left[\begin{array}{cc}
\operatorname{Cov}(\mathbf{C}(0)) & 0 \\
0 & S_{d}
\end{array}\right]
$$

\section{EXPERIMENTAL RESULTS}

We applied the proposed EKF framework to the pharmacokinetic analysis of ICG data obtained from four Fischer rats with adenocarcinoma. R3230ac, adenocarcinoma cells were injected below the skin into four Fischer rats, 3 weeks prior to measurements. ${ }^{8}$ Contrast agent experiments were conducted on four of these rats just before sacrifice. Tumor sizes for these rats ranged in diameter from 5 to $30 \mathrm{~mm}$. The ICG concentration data is collected with an MRI-NIR imager. The configuration of the apparatus and the detailed data collection procedure have been reported in. ${ }^{29,30}$

Figure 4 presents ICG concentrations $(\mu M)$ from four different rats. Tumors in Rat 1 and 2 are classified as necrotic because of low tissue oxy-hemoglobin, low total hemoglobin, and low Gd-DTPA enhancement levels. Tumors in Rat 3 and 4 are classified as edematous due to high water content. It can be observed from Figure 4 that the necrotic cases display low peak ICG concentration values and slowly rising slopes unlike the edematous cases with high peak values and sharp rising slopes. ${ }^{8,31}$

In order to characterize the difference between these two tumors, we estimated the pharmacokinetic parameters $k_{i n}, k_{\text {out }}$ and $k_{\text {elm }}$, and the volume fractions $v_{p}$ and $v_{e}$ for each rat. The estimated parameters are tabulated in Table 1 . The values of $k_{\text {out }}$ range from 0.0105 to $0.0777 \mathrm{sec}^{-1}$ and the values of $k_{\text {in }}$ range from 0.0249 to 0.0840 $\mathrm{sec}^{-1}$. Note that the permeability rates for necrotic cases are lower than the ones observed for the edematous 


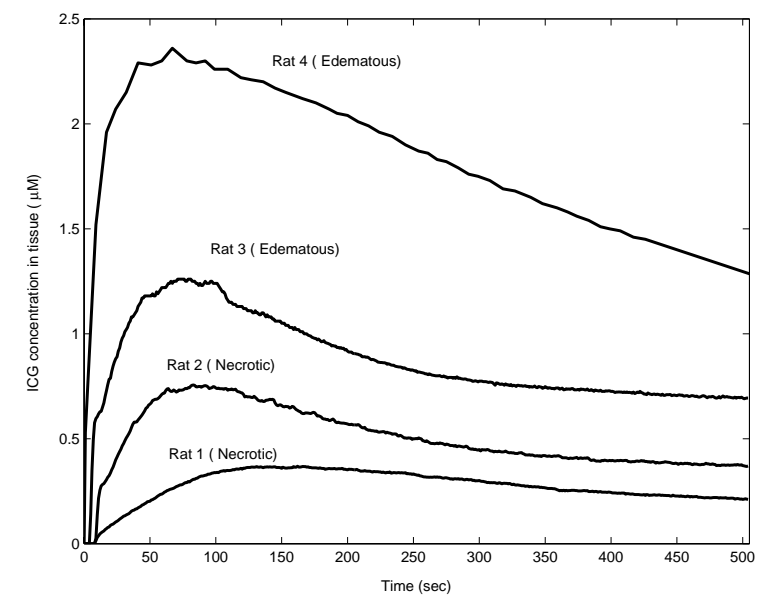

Figure 4. ICG concentrations measured in tissue for four different rats.

Table 1. Estimated pharmacokinetic parameters using EKF algorithm

\begin{tabular}{cccccc} 
& $k_{\text {in }}\left(\sec ^{-1}\right)$ & $k_{\text {out }}\left(\sec ^{-1}\right)$ & $k_{\text {elm }}\left(\sec ^{-1}\right)$ & $v_{e}$ & $v_{p}$ \\
\hline Rat 1 (Necrotic) & 0.0249 & 0.0105 & 0.00464 & 0.167 & 0.0142 \\
Rat 2 (Necrotic) & 0.0354 & 0.0298 & 0.00483 & 0.254 & 0.0242 \\
Rat 3 (Edematous) & 0.0690 & 0.0493 & 0.00395 & 0.304 & 0.0484 \\
Rat 4 (Edematous) & 0.0840 & 0.0777 & 0.00402 & 0.530 & 0.0703 \\
\hline
\end{tabular}

cases. These results indicate that the permeability rates are higher in advanced tumors. Additionally, the estimated values for the pharmacokinetic rates are much higher than the normal tissue values due to the increased leakiness of the blood vessels around the tumor region. ${ }^{8,34}$ The estimated plasma volume fractions agrees with the values reported earlier, ${ }^{8}$ and the values presented in literature. ${ }^{32,33}$ These results confirm that $v_{p}$ can be significantly large in tumors and that $v_{p}$ increases depending on the stage of the tumor. ${ }^{20}$ The estimated values of EES volume fractions, $v_{e}$, range from 0.167 to 0.530 agreeing with a level of 0.2 to 0.5 reported before. ${ }^{19}$ Note that these results are valid only for the ICG pharmacokinetics in tumor cells R3230ac, adenocarcinoma and may not be generalized for other types of contrast agents or tumor types.

Figure 5 shows the estimated ICG concentrations in the plasma and the EES compartments for Rats 1 to 4. Note that initial estimates of concentrations are noisy due to the limited data used in the recursive EKF estimation. This can be improved by backward smoothing. The peak values of the plasma concentration, $C_{p}$, range from $1.84 \mu M$ to $4.28 \mu M$. The absolute value of the concentrations may not be very useful. However, concentration of ICG in one compartment relative to the concentration in another compartment may provide useful information. We consider the ratio of the peak concentrations in the plasma and EES as a potential parameter to discriminate different tumors. The peak $C_{p} / C_{e}$ ratio for Rats 1 to 4 is $0.454,0.593,0.787,1.151$, respectively. This ratio is higher in edematous cases agreeing with the fact that, ICG-albumin leaks more to the EES in advanced tumor. Additionally, the ICG concentration in plasma decays faster than the ICG concentration the in EES due to ICG elimination through liver and kidneys.

\section{CONCLUSION}

In this paper, we present a Kalman filtering framework for the modeling and estimation of ICG pharmacokinetics. A two-compartmental model is fitted to the rat data using EKF estimation methodology. The pharmacokinetic 

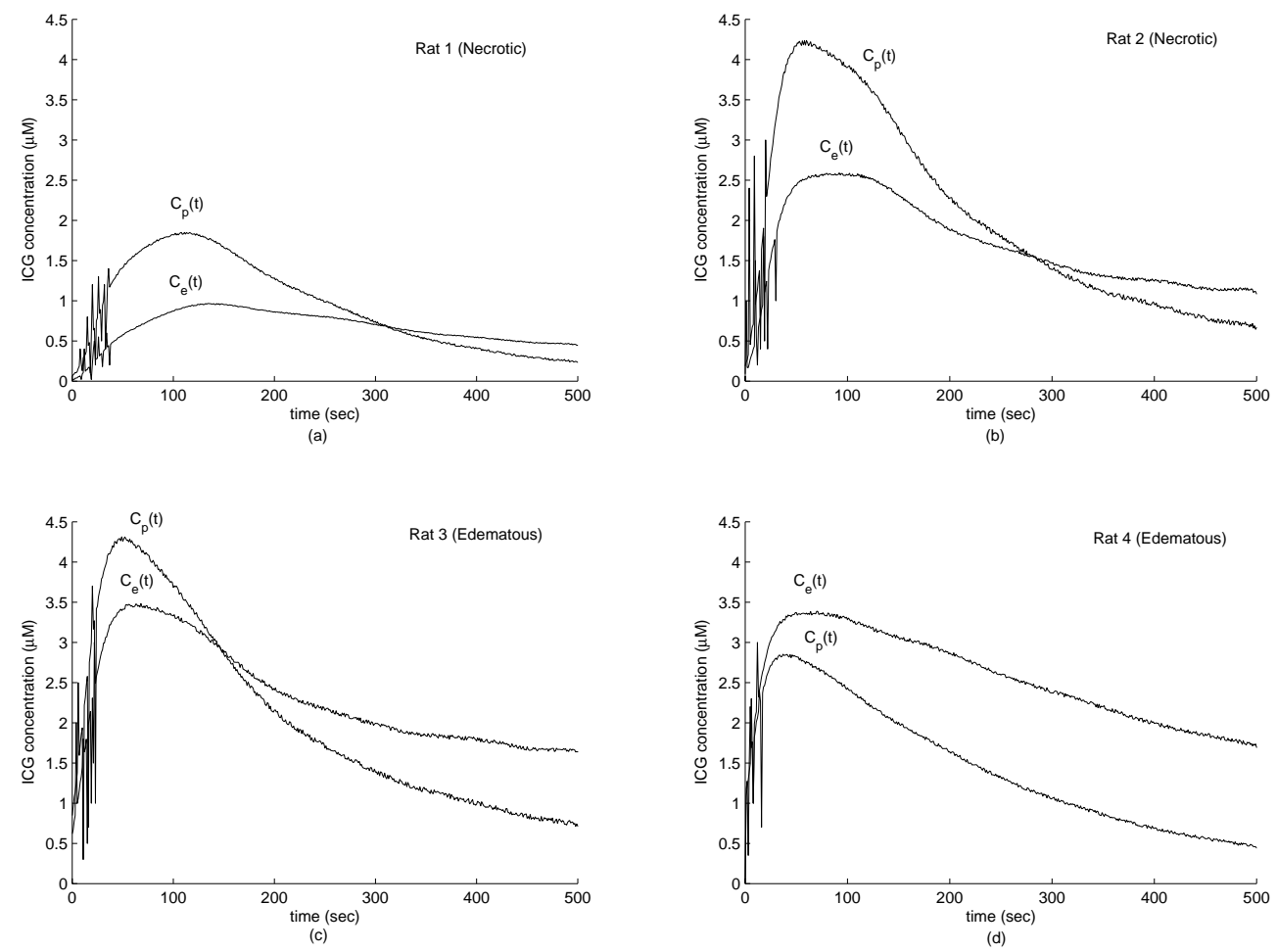

Figure 5. ICG concentrations in plasma, $C_{p}(t)$ and EES, $C_{e}(t)$, for four different rats. $5(\mathrm{a})$ for Rat $1,5(\mathrm{~b})$ for Rat 2 , 5 (c) for Rat 3, 5(d) for Rat 4 . .

parameters and volume fractions are estimated for different compartments. The results indicate that the permeability rates are higher for advanced tumors. Additionally, we estimated the ICG concentrations in different compartments. The concentrations in different concentrations may provide additional parameters for tissue characterization. As a future work, we plan to apply EKF framework to different optical contrast agents collected from animal subjects and to the ICG data collected from human subjects.

\section{ACKNOWLEDGEMENT}

This work was supported by U.S. Army Medical Research Acquisition Activity under grant W 81XWH-04-1-0559, and Office of Naval Research under grant N 000014-04-1-0694.

\section{REFERENCES}

1. B. Chance, R.R. Alfano, B.J. Tromberg, M. Tamura and E.M. Sevick-Muraca, eds, "Optical tomography and spectroscopy of tissue IV," Proc. 4250 SPIE-The International Society for Optical Engineering, Bellingham, WA, 2001.

2. A. Yodh and B. Chance, "Spectroscopy and imaging with diffusing light," Phys. Today Vol. 48-3, pp. 34-40, 1995.

3. S. Solin, "Optical imaging of breast tumor by means of continuous waves," Adv. Exp. Med. Biol. Vol. 411, pp. 227-232, 1997.

4. V. Ntziachristos, X.H. Ma, A.G. Yodh and B. Chance, "Multichannel photon counting instrument for spatially resolved near infrared spectroscopy," Rev Sci Instrum, Vol. 70-1, pp. 193201, Part 1, 1999. 
5. D. Hawrys and E. Sevick-Muraca, "Developments toward diagnostic breast cancer imaging using NearInfrared optical measurements and fluorescent contrast agents," Neoplasia Vol.2, pp. 388417, 2000.

6. Furukawa, K., D. H. Crean, T. S. Mang, H. Kato and T. J. Dougherty (1995) "Fluorescence detection of premalignant, malignant, and micrometastatic disease using hexylpyropheophorbide," Proc. SPIE Vol. 2371, pp. 510514.

7. M. Gurfinkel, A. B. Thompson, W. Ralston, T. L. Troy, A. L. Moore, T. A. Moore, J. D. Gust, D. Tatman, J. S. Reynolds, B. Muggenburg, K. Nikula, R. Pandey, R. H. Mayer, D. J. Hawrysz, and E. M. Sevick-Muraca, "Pharmacokinetics of ICG and HPPH-car for the detection of normal and tumor tissue using fluorescence, near-infrared reflectance imaging: a case study," Photochem. Photobiol., Vol. 72, 2000, pp. 94-102.

8. D.J. Cuccia, F. Bevilacqua, A. J. Durkin, S. Merritt, B. J. Tromberg, G. Gulsen, H. Yu, J. Wang, and O. Nalcioglu, "In vivo quantification of optical contrast agent dynamics in rat tumors by use of diffuse optical spectroscopy with magnetic resonance imaging coregistration," Applied Optics, Vol. 42, No 1, June 2003.

9. X. Intes, J. Ripoll, Y. Chen, S. Nioka, A. G. Yodh, B. Chance, "In vivocontinuous-wave optical breast imaging enhanced with Indocyanine Green," Med. Phys. Vol. 30-6, June 2003.

10. D. Hansen, A. Spence, T. Carski, and M. Berger, "Indocyanine green (ICG) staining and demarcation of tumor margins in a rat glioma model," Surg. Neurol. Vol. 40, pp. 451-456, 1993.

11. H. Shinohara, A. Tanaka, T. Kitai, N. Yanabu, T. Inomoto, S. Satoh, Hatano, Y. Yamaoka, and K. Hirao, "Direct measurement of hepatic Indocyanine Green clearance with near-infrared spectroscopy: separate evaluation of uptake and removal," Hepatology Vol. 23, pp. 137-144, 1996.

12. A. ElDeosky, A. Seifalian, M. Cope, D. Delpy, and B. Davidson, "Experimental study of liver dysfunction evaluated by direct Indocyanine green clearance using near infrared spectroscopy," Br. J. Surg. Vol. 86, pp. 1005-1011, 1999.

13. X. Li, B. Beauvoit, R. White, S. Nioka, B. Chance, and A. Yodh, "Tumor localization using fluorescence of Indocyanine Green (ICG) in rat models," Proc. SPIE Vol. 2389, pp. 789-797, 1995.

14. M. S. Yates, C. J. Bowmer, and J. Emmerson, "The plasma clearance of indocyanine green in rats with acute renal failure: effect of dose and route of administration," Biochem. Pharmacol. Vol. 32, pp. 3109-3114, 1983.

15. C. W. Tornoe, "Grey-Box PK/PD Modeling of Insulin," M.S. Thesis, June 28, 2002.

16. D. H. Anderson, Lecture Notes in Biomathematics, Springer-Verlag, Berlin, 1983.

17. J. A. Jacquez, Compartmental analysis in biology and medicine, Kinetics of distribution of tracer-labeled materials, Elsevier Pub. Co., New York, 1972.

18. C. Cobelli, D. Foster, and G. Toffolo, Tracer kinetics in biomedical research: from data to model, Kluwer Academic/Plenum, New York, c2000.

19. P. S. Tofts, DPhil, G. Brix, D. L. Buckley, J. L. Evelhoch, E. Henderson, M. V. Knopp, H. B.W. Larsson, T. Lee, N. A. Mayr, G. J.M. Parker, R. E. Port, J. Taylor, and R. M. Weisskoff, "Estimating Kinetic Parameters From Dynamic Contrast-Enhanced T1-Weighted MRI of a Diffusable Tracer: Standardized Quantities and Symbols," Jour. Mag. Res. Ima. Vol. 10, pp. 223232, 1999.

20. P. S. Tofts, "Modeling tracer kinetics in dynamic Gd-DTPA MR imaging," J. Magn. Reson. Imag. Vol. 7, pp. 91-101, 1997.

21. C. Chen, Linear System Theory and Design, Oxford University Press, New York, 1999.

22. P. Zarchan, Fundamentals of Kalman filtering : a practical approach, American Institute of Aeronautics and Astronautics, Reston, 2000.

23. C.K.Chui, G. Chen, Kalman Filtering with real time applications, Springer, Berlin, 1999.

24. C. E. Catlin, Estimation, control, and the discrete Kalman filter, Springer, New York, 1989.

25. L. Ljung, "Asymptotic Behavior of the Extended Kalman Filter as a Parameter Estimator for Linear Systems," IEEE Tran. Automa. Control, Vol. AC-24, No. 1, pp. 36-50, Feb 1979.

26. R. Togneri, and L. Deng, "Joint State and Parameter Estimation for a Target-Directed Nonlinear Dynamic System Model," IEEE Tran. on Sig. Proc, Vol. 51, No. 12, pp. 3061-3070, Dec 2003.

27. L. Nelson, and E. Stear, "The Simultaneous On-Line Estimation of Parameters and States in Linear Systems," IEEE Tran. on Auto. Control, pp. 94-98, Feb 1976.

28. B. F. La Scala, and R. R. Bitmead, "Design of an extended Kalman filter frequency tracer," IEEE Tran. on Sig. Proc, Vol. 44, No. 3, pp. 739-742, March 1996. 
29. F. Bevilacqua, A. J. Berger, A. E. Cerussi, D. Jakubowski, and B. J. Tromberg, "Broadband absorption spectroscopy in turbid media by combined frequency-domain and steady-state methods," Appl. Opt., Vol. 39, pp. 64986507, 2000.

30. D. J. Jakubowski, "Development of broadband quantitative tissue optical spectroscopy for the non-invasive characterization of breast disease," Beckman Laser Institute, University of California, Irvine, Irvine, Calif., 2002.

31. S. Merritt, F. Bevilacqua, A. J. Durkin, D. J. Cuccia, R. Lanning, B. J. Tromberg, G. Gulsen, H. Yu, J. Wang, and O. Nalcioglu, "Monitoring tumor physiology using near-infrared spectroscopy and MRI coregistration," Appl. Opt. Vol. 42, pp. 2951-2959, 2003.

32. J. B. Fishkin, O. Coquoz, E. Anderson, M. Brenner, and B. J. Tromberg, "Frequency-domain photon migration measurements of normal and malignant tissue optical properties in a human subject," Appl. Opt., Vol. 36, pp. 1020, 1997.

33. D. L. Buckley, "Uncertainty in the analysis of tracer kinetics using dynamic contrast-enhanced T1-weighted MRI," Magn. Reson. Med., Vol. 47, pp. 601606, 2002.

34. M. Y. Su, A. Muhler, X. Lao, and O. Nalcioglu, "Tumor characterization with dynamic contrast-enhanced MRI using MR contrast agents of various molecular weights," Magn. Reson. Med., Vol. 39, pp. 259269, 1998. 